nous l'avons constaté le jour du départ de l'accouchée quand, la fatigue augmentant, la matière grasse du lait et sa quantité diminuaient. Nous observerons le même phénomène dans l'hypogalactie constitutionnelle.

Les inflammations aiguës du sein et leurs effets sur la qualité du lait seront étudiés dans un chapitre spécial.

(A suivre.)

\title{
L'UTILISATION DE L'ACIDE LACTIQUE
}

\author{
par \\ G. GÉNIN \\ Ingénieur E. P. C.
}

Dans le compte rendu que nous avons donné des travaux du Congrès du Printemps de l'American Chemical Society, qui s'est tenu à Baltimore, en avril 1939, nous avons résumé en quelques mots la très intéressante communication de L. T. Sмгін et H. V. Claborn sur l'utilisation de l'acide lactique. L'importance de cette question mérite que la communication de ces deux auteurs soit analysée d'une façon plus complète.

Depuis longtemps déjà, les chimistes et les techniciens appartenant à l'industrie laitière, songeaient que cette dernière pourrait retirer des bénéfices appréciables de l'utilisation d'un grand nombre de produits provenant du sérum du lait et en particulier du lactose. On sait que par fermentation du lactose, on obtient de l'acide lactique et les deux auteurs américains se sont proposés de montrer quelles pouvaient être-les applications industrielles de l'acide lactique et de ses dérivés.

Le lactose ou sucre du lait qui a la formule $\mathrm{C}^{12} \mathrm{H}^{22} \mathrm{O}^{11}$ existe dans le lait de tous les mammifères terrestres. Le lait de vache en particulier contient environ $5 \%$ de lactose. Il est done facile de voir quelle quantité énorme de lactose pourrait être produite par utilisation soit du lait écrémé, soit du petit-lait et du sérum provenant de la fabrication du beurre, du fromage, etc.

La fermentation du lactose en acide lactique est une opération aujourd'hui industrialisée et, aux Etats-Unis, il existe déjà une usine qui utilise le lactose du petit-lait en vue de sa transformation en acide lactique. D'autres installations emploient dans le même but d'autres matières premières comme les mélasses et le sucre retiré du maïs. On estime, d'après les derniers renseignements statistiques, qu'il est produit chaque année aux Etats-Unis environ 5 millions de pounds d'acide lactique à $100 \%$.

(1) Voir " Le Lait ", n* 188, septembre-octobre 1939, p. 891. 
L'acide lactique ou acide alpha-hydroxypropionique qui répond à la formule $\mathrm{CH}^{3} \mathrm{CHOH} \mathrm{COOH}$ a été découvert dans le lait sur par Scheele, en 1780. Si les produits contenant du lait sur et par conséquent de l'acide lactique sont fabriqués depuis fort longtemps sur une échelle industrielle, par contre la préparation et l'isolement de l'acide lactique ne sont effectués commercialement que depuis environ trois ans.

On trouve aux Etats-Unis au moins quatre qualités commerciales d'acide lactique qui sont: l'acide lactique brut dilué, l'acide lactique comestible à $50 \%$, la qualité plastique et l'acide à $85 \%$ répondant aux spécifications de la pharmacopée américaine. Que ce soit sous l'une ou sous l'autre de ses formes, l'acide lactique a déjà des applications très variées et importantes, par exemple dans la fabrication des produits pharmaceutiques et comme acidulant dans la fabrication des produits alimentaires : essence de fruits, extraits, sirops, levure, crèmes glacées, pickles, etc.

On a également utilisé cet acide pour la fabrication des eaux gazeuses et limonades à la place de l'acide citrique, il a été employé dans la teinture pour le rouge turc, dans la teinture des soies et autres textiles, comme mordant pour les impressions sur calicot, pour le traitement des plumes, pour le traitement des peaux et dans le tannage végétal. Dans la préparation des résines phénoliques coulables, on l'utilise comme catalyseur et plastifiant.

Lorsque l'acide lactique est déshydraté par la chaleur, il se forme de l'acide monolactyl-lactique de formule

\section{$\mathrm{C}^{3} \mathrm{HCH}(\mathrm{OH}) \mathrm{COOCH}\left(\mathrm{CH}^{3}\right) \mathrm{COOH}$}

de l'acide dilactyl-lactique, de l'acide trilactyl-lactique, une lactide et finalement des produits fortement condensés et polymérisés. Ces derniers sont insolubles dans l'eau et dans les alcools dilués, ils peuvent être employés dans la préparation de vernis destinés à être cuits au four.

Ainsi que sa formule l'indique, l'acide lactique possède deux groupes $\mathrm{OH}$ actifs, l'un est le groupe carboxyle normal, et l'autre en position alpha est un groupe alcool secondaire. Il est par conséquent possible d'obtenir en partant d'acide lactique des sels bimétalliques, des éthers, des esters et diverses combinaisons de ces produits.

Les sels métalliques industriels s'obtiennent en neutralisant au moyen de sels alcalins l'acide lactique tel qu'il se forme dans les récipients où s'effectue la fermentation du lactose. Lorsque la neutralisation est terminée, les solutions sont concentrées et les sels de l'acide lactique cristallisent. Lorsqu'on dásire obtenir des sels purs, il faut raffiner les sels bruts, et employer une méthode 
assez longue de purification. Le lactate de calcium se prépare facilement et jusqu'ici, e'est le sel de l'acide lactique qui est le plus important. Il est employé, en médecine, dans les poudres destinées à faciliter la cuisson du pain, etc. On a récemment utilisé le sel de sodium à la place de la glycérine, car ce produit a l'avantage d'absorber et de retenir l'humidité, on l'ajoute donc au tabac pour éviter le séchage de ce dernier. Des brevets signalent également l'emploi du lactate de cuivre dans la préparation de bains électrolytiques. Le sel de zinc est enfin employé parfois dans la préparation de l'acide lactique pur.

Les éthers de l'acide lactique qui répond snt à la formule $\mathrm{CH} \mathrm{CH}(\mathrm{OR}) \mathrm{COOH}$ peuvent s'obtenir par action des halogénures ou des sulfates d'aleoyle sur les dérivés métalliques. D'une façon générale, ils sont plus difficiles à préparer que les mono et les diesthers. Les éthers sont insolubles dans l'eau et on peut les utiliser comme solvants, comme plastifiants, ainsi que comme produits destinés à modifier les propriétés des laques, vernis, matières plastiques et encres d'imprimerie.

Les esters répondant à la formule générale $\mathrm{CH}^{3} \mathrm{CHOH} \mathrm{COOR}$ s'obtiennent facilement par réaction de l'acide lactique avec un alcool en présence d'un acide minéral comme les acides sulfurique, chlorhydrique ou phosphorique, jouant le rôle de catalyseur. Le rendement en esters varie de 60 à près de $100 \%$, il dépend essentiellement de la méthode que l'on emploie pour l'élimination de l'eau au fur et à mesure de sa formation au cours de la réaction. Les esters inférieurs, e'est-à-dire les esters méthylique, éthylique et propylique, sont solubles dans l'eau et ont tendance à s'hydrolyser; aussi ces caractéristiques tendent à limiter leur emploi.

Les esters supérieurs : esters butylique, amylique, laurylique, stéarylique, etc., sont insolubles dans l'eau et plus stables. Tous ces esters sont susceptibles d'être utilisés comme solvants, plastifiants, ainsi que pour la modification des caractéristiques des laques, matières plastiques, vernis, colles, encres, etc.

Le groupe hydroxy en position alph a de ces esters peut également réagir facilement avec les anhydrides d'acides pour former les esters correspondants. Un exemple est donné par la préparation de l'alpha-acétoxypropionate de méthyle. Pendant cette réaction, la formation de l'ester a lieu presque quantitativement. Les esters ainsi obtenus sont insolubles dans l'eau et ont les mêmes applications que les précédents.

Par pyrolyse, ces diesters de l'acide lactique peuvent être transformés en dérivés de l'acide acrylique correspondant. C'est ainsi par exemple que l' $\alpha$-acétoxypropionate de méthyle est décomposé en acrylate de méthyle monomère $\left(\mathrm{CH}^{2}=\mathrm{CH} \mathrm{COOCH}^{3}\right.$ et en acide 
acétique. Ce monomère se polymérise facilement sous l'action de la chaleur ou en présence de catalyseur constitué par des peroxydes et on obtient de l'acrylate de polyméthyle. Des essais de laboratoire ont déjà montré que l'acrylate de polyméthyle obtenu de cette façon peut être fourni à un prix beaucoup plus avantageux que le polymère préparé par le procédé actuellement employé dans l'industrie et dans lequel on part de cyanhydrine.

Les acrylates monomères présentent d'ailleurs un domaine d'application extrêmement vaste. On peut par exemple les copolymériser en même temps que les esters méthacryliques, les composés de vinyle, le butadiène et le styrène. Ces copolymères ainsi obtenus sont des produits qui sont susceptibles de se mouler par injection, dont les caractéristiques physiques sont excellentes et qui peuvent être également livrés sous forme de baguettes, de tubes et de feuilles. Les produits ainsi préparés sont bien supérieurs aux polymères auxquels on a ajouté des plastifiants.

Lorsque l'acide acrylique est polymérisé, i. e produit un polymère qui prend la forme d'une masse croûteuse. Les esters acryliques se polymérisent également et au cours de cette transformation, on constate une élévation de la viscosité et la formation d'abord d'un liquide visqueux, puis de substances plus ou moins élastiques et transparentes. Ces esters polyacryliques sont insolubles dans l'eau et dans l'alcool, mais ils se dissolvent dans les esters acryliques monomères, dans les solvants aromatiques (benzène, toluène), dans les cétones (acétone, méthyléthylcétone) et dans les hydro-carbures halogénés (chloroforme, dichlorure d'éthylène). Ces esters polvacryliques se sont révélés comme des produits particulièrement intéressants par suite de leur limpidité, de leur élasticité, de leurs bonnes propriétés mécaniques, de la facilité avec laquelle ils se dissolvent et de leur stabilité à la lumière, ils trouveront certainement de très importants débouchés dans l'industrie des vernis, des laques, des compounds d'imprégnation et des films. On peut également imprégner avec ces polymères tous les textiles, ainsi que le papier, sans qu'il soit nécessaire de faire subir aux produits imprégnés une cuisson ou tout autre traitement particulier. Par cette imprégnation, les tissus et les papiers peuvent être rendus résistants à l'humidité et imperméables aux gaz.

Ces quelques exemples montrent donc le rôle important que sont susceptibles d'être appelés à jouer l'acide lactique et ses nombreux dérivés dans le domaine des solvants, des plastifiants, des vernis, des matières plastiques, des encres, sans compter les applications dans le domaine des produits alimentaires. 